\title{
Criminologie
}

\section{Autour de Croissance et décroissance du crime}

\section{Daniel Élie et Maurice Cusson}

Volume 25, numéro 1, 1992

Les femmes et le contrôle pénal, questions féministes

URI : https://id.erudit.org/iderudit/017319ar

DOI : https://doi.org/10.7202/017319ar

Aller au sommaire du numéro

Éditeur(s)

Les Presses de l'Université de Montréal

ISSN

0316-0041 (imprimé)

1492-1367 (numérique)

Découvrir la revue

Citer ce document

Élie, D. \& Cusson, M. (1992). Autour de Croissance et décroissance du crime. Criminologie, 25(1), 135-147. https://doi.org/10.7202/017319ar

\section{Résumé de l'article}

Deux professeurs de l'École de criminologie de l'Université de Montréal réfléchissent sur l'évolution et les fluctuations de la criminalité. Leur débat se situe principalement autour des idées émises par Maurice Cusson dans l'une de ses plus récentes publications. Daniel Élie engage la discussion à laquelle répond ensuite l'auteur. d'utilisation que vous pouvez consulter en ligne.

https://apropos.erudit.org/fr/usagers/politique-dutilisation/ 


\begin{abstract}
Deux professeurs de l'École de criminologie de l'Université de Montréal réfléchissent sur l'évolution et les fluctuations de la criminalité. Leur débat se situe principalement autour des idées émises par Maurice Cusson dans l'une de ses plus récentes publications. Daniel Élie engage la discussion à laquelle répond ensuite l'auteur.
\end{abstract}

\title{
1. RÉFLEXION SUR LES PROPOSITIONS THÉORIQUES DANIEL ÉLIE
}

L'intérêt pour l'évolution du nombre de crimes connus est au cœur des préoccupations criminologiques. En fait, répondre aux questions qu'elle soulève est un des buts de la discipline. Il s'est dit beaucoup de choses durant la période de croissance rapide du nombre de crimes entre 1965 et 1975 . On constate que le sujet se représente avec insistance depuis le constat affiché d'une stabilisation voire d'une diminution de certains types d'incidents depuis les cinq dernières années en Amérique du Nord. Les mêmes thèses sont reprises et reconsidérées par les chercheurs (démographie, classe d'âge, opportunité, situation économique, etc.). Chacun veut analyser au mieux cette conjoncture propice à la mise à l'épreuve de plusieurs propositions théoriques et tout texte sur le sujet devient visible et soulève l'intérêt.

C'est dans ce contexte que $\mathrm{M}$. Maurice Cusson a publié récemment un livre intitule Croissance et décroissance du crime (PUF, 1990). Après y avoir exposé un grand nombre de faits susceptibles d'éclairer les fluctuations des taux de criminalité, on y présente un groupe de propositions qui constituent, selon l'auteur, une nouvelle théorie formelle (au sens de Boudon) susceptible d'expliquer les variations du phénomène qui nous intéresse. Ce texte nous fournit l'occasion de faire avancer le débat.

1. CUSSON, Maurice (1990), Croissance et décroissance du crìme, P.U.F., Paris.

2. Professeur agrégé, École de criminologie, Université de Montréal, C.P. 6128, succ. " A ", Montréal (Québec) H3C 3J7.

3. Directeur, École de criminologie, Université de Montréal, C.P. 6128, succ. «A ", Montréal (Québec) H3C 3J7. 
Cette théorie est résumée comme suit, selon les termes de l'auteur :

La croissance des opportunités stimule celle du crime cependant que le contrôle social et la sanction pénale agissent comme des freins. Les progrès du crime encouragent l'autoprotection, laquelle, en réduisant les opportunités, fait reculer la criminalité. Sous la pression de la montée du nombre des affaires à traiter, un système pénal stagnant doit en évacuer le surplus, ce qui, par la baisse de la certitude de la peine qui s'ensuit, favorise une nouvelle croissance du crime. $\mathrm{Si}$, par contre, la probabilité qu'un crime soit sanctionné augmente, la criminalité baissera, ce qui décongestionnera l'appareil pénal et rendra possible un mouvement de croissance de la certitude de la peine, etc. (p. 142).

La théorie formulée est supposée reposer sur les observations faites par l'auteur dans le domaine de l'application des lois, de la démographie, des opportunités, etc. Il est donc intéressant et important de considérer l'exactitude des données utilisées et leur relation avec le développement des criminalités. Il est également opportun d'analyser le statut et la valeur de la théorie soumise. Notre intérêt réside, à ce point, dans la question de savoir s'il est véritablement possible d'élaborer sur ce sujet une théorie formelle globale dans notre domaine.

Examinons d'abord les faits. On constate en premier lieu que, tout au long du texte, il est question du crime ou de la criminalité, au singulier. On se trouve ainsi à rassembler dans une dynamique commune des faits aussi divers que la fraude, le viol, les fausses alertes à la bombe ou l'homicide. Or, il faut bien convenir qu'il s'agit là de faits suffisamment différents pour qu'on ne puisse les aborder avec une seule et même méthode d'analyse. L'agrégation des types d'incidents introduit de la confusion. On conçoit mal, au stade actuel de nos connaissances, qu'une même théorie puisse s'appliquer au meurtre et à la fraude au moyen de cartes de crédit. Il est possible de faire certains emprunts à la science économique, notamment les concepts d'offre et de demande, d'inélasticité de la demande dans certains marchés, pour éclairer l'évolution de faits tels que les vols d'automobiles ou le trafic de cocaïne. Cela se pratique couramment depuis que les économistes portent un intérêt à notre discipline (par exemple, A. T. Rogers III, 1973). Par contre, la même perspective théorique sera peu pertinente dans le cas d'incidents tels que le vol à main armée ou l'homicide. $\mathrm{Ne}$ pas tenir compte de cette réalité peut conduire à des formulations trop générales et peu éclairantes. Mais nous nous interrogeons également sur la présentation même de l'ensemble du phénomène. Sur ce point, le malaise vient du niveau d'agrégation spatiale. On peut réellement se 
demander s'il est raisonnable, en l'état actuel de nos connaissances, de parler encore d'un type de criminalité pour l'ensemble du territoire américain ou canadien. Nous posons que le niveau d'agrégation spatiale est trop élevé dans ce cas et nous nous expliquons brièvement sur ce point.

L'observation des séries chronologiques décrivant les fluctuations de plusieurs types de criminalité dans différents territoires, ou portions de territoires, éclaire très vite l'état des choses. On note immédiatement la diversité des modes d'évolution d'une catégorie de crime, durant une même période, selon les zones étudiées. Cette variété est sensiblement (totalement ?) occultée lorsqu'on traite du même phénomène à un niveau d'agrégation des données impliquant l'ensemble du territoire nord-américain. Cependant, on constate que les discussions et propositions théoriques rapportées dans la première partie de ce texte s'appuient sur ce dernier type de données. Or, l'expérience révèle que les relations observées à un niveau ne sont pas nécessairement transférables ou généralisables à un autre. La corrélation entre un facteur quelconque et l'évolution d'un type de crime varie selon l'étendue et les caractéristiques du territoire dans lequel les deux variables sont observées. Par contre, elle peut demeurer constante dans une même unité territoriale. Ces faits, constamment observés dans les rapports de recherche et les publications en criminologie, suscitent des doutes importants au sujet de l'utilité et de la crédibilité d'une théorie formelle générale sous la forme qu'on nous propose ici.

Dans un premier temps, donc, on est amené à penser que pour l'analyse des conjonctures usuelles, c'est-à-dire celle qui s'applique à des agglomérations urbaines telles que la Communauté urbaine de Montréal ou la ville de Toronto, celle qui intéresse directement les administrations publiques, des propositions issues d'observations globales faites sur l'ensemble d'un territoire aussi vaste et diversifie que les États-Unis ou le Canada ont tout au plus valeur d'indice. L'espérance en gain pour l'explication, la prédiction ou la compréhension demeure cependant faible.

En effet, lorsqu'on ne tient pas compte de la variété des profils d'évolution dans les différentes sous-sections d'un territoire, on ne connaît pas vraiment la nature ou l'ampleur d'un changement signalé par des données décrivant l'ensemble de ce territoire. Ainsi, les propositions concernant cet ensemble, en plus de n'être pas généralisables, ont de fortes chances de s'avérer fausses dans une section ou un sous- 
ensemble particulier. Ceci représente un problème majeur du discours produit dans ce livre.

Par ailleurs, l'auteur affirme (p. 105) que, selon toute vraisemblance, la réaction pénale a subi une érosion entre 1960 et 1980, ce qui expliquerait l'augmentation de la criminalité durant cette période puisqu'il existerait, semble-t-il, un lien direct entre les deux faits (les mécanismes sociaux de l'impunité, p. 110; la dynamique de l'érosion pénale, p. 117).

Ce genre de proposition est certainement le résultat d'une lecture très sélective (ou partiale ?) des faits, des données statistiques largement disponibles et des résultats de recherches connus. Sur ce point, il convient de souligner deux faits. Le premier est que la proposition est fausse. Selon les données fournies par le ministère du Solliciteur général du Canada (1984) de 1950 à 1982, en chiffres absolus, la population dans les pénitenciers a doublé, avec une stabilisation entre 1966 et 1970. Suivant le taux par 100000 habitants, il est demeuré stable depuis 1968. Le système fonctionne à pleine capacité. On peut difficilement dans ces conditions parler d'une érosion de la peine au Canada, surtout lorsqu'on tient compte de l'évolution importante de la population en liberté surveillée en plus de celle des prisons et pénitenciers. Aux États-Unis, les données officielles signalent le même fait (voir NCCD focus, avril 1988). On y constate que les taux d'incarcération présentent un accroissement soutenu de 1850 à 1940, une diminution marquée pendant la dernière guerre, un plateau de 1950 à 1970 , et une augmentation exponentielle à partir de cette année. Un texte de Blumstein (1988) est également très éclairant sur le sujet. La même situation prévaut en Angleterre et au pays de Galles, c'est-à-dire un accroissement continu du taux d'incarcération. Non, il n'y a pas d'érosion observable de la peine dans ces données statistiques.

Le deuxième point à relever est que la relation entre les deux faits est loin d'être claire et systématique. Il s'agit d'un vieux débat en criminologie et les résultats des recherches sur le sujet sont assez connus. On ne peut pas affirmer de science certaine qu'une variation du taux d'incarcération implique une variation équivalente ou concomitante du nombre de crimes. En fait, la littérature sur le sujet nous révèle que des chercheurs tels que W. J. McGuire et R. G. Sheeham (1983) trouvent une relation entre les deux faits mais qu'un groupe assez important de travaux constatent le contraire (Bowker, 1981 : Biles, 1983 ; Pontell, 1979). En fait, ce dernier auteur remarque qu'on peut penser que dans certains pays et durant certaines périodes le taux de criminali- 
té peut influencer plus fortement la certitude de la peine que la certitude de la peine influence le taux de criminalité. On ne se méfiera jamais assez dans ce domaine des systèmes de raisonnement impliquant une causalité simple et réversible. Les résultats de recherche soumis par Tittle et Row (1974) appuient l'argument de la dissuasion. Ils soulignent même que la certitude de la peine doit atteindre un certain niveau $(30 \%)$ avant de faire la preuve de son efficacité. Par contre, ces auteurs signalaient déjà que l'ordre causal, dans les corrélations obtenues, demeure un problème difficile à résoudre. De plus, ils ont constaté des corrélations très variables en valeurs absolues selon le niveau d'agrégation spatiale. Ils concluent qu'il reste à développer les connaissances empiriques au sujet des conditions dans lesquelles des sanctions pénales sont plus ou moins importantes.

Durant la même période, Wellford (1974) rapporte que les relations entre la dissuasion, la certitude de la peine et le nombre de crimes signalés sont relativement faibles et peu significatives. Les corrélations observées sont de l'ordre de $-0,15$ à $-0,25$.

Pendant longtemps, il a été difficile, dans le cadre des recherches sur le sujet. de tenir compte des délais de réaction de la population de prédateurs de toutes sortes au mouvement du système pénal. Les récents développements des méthodes quantitatives ont levé ce handicap. Ainsi, Chamblin (1988) a utilisé la technique ARIMA (AutoRegressive Integrated Moving Average) pour analyser la relation entre les fluctuations dans le volume des arrestations et celle de la criminalité en Oklahoma. Dans une étude fine et détaillée retenant les variations mensuelles des deux faits, il arrive à la conclusion que les deux systèmes semblent être en relation d'indifférence puisque aucune variété du modèle n'a permis d'observer une corrélation significative. Il souligne également la possibilité que la population de prédateurs ne soit pas aussi rationnelle dans son comportement, comme on semble le supposer souvent, et qu'elle ne percevrait pas nécessairement bien les risques encourus dans le cours de leurs activités illégales.

Le cas le plus frappant nous est fourni par la comparaison faite par D. Biles (1983) entre la Grande-Bretagne et l'Australie pour une période allant de 1960 à 1980. Dans les deux cas, le nombre de crimes déclarés suit un accroissement soutenu et du même type. On constate qu'en Grande-Bretagne, le taux d'incarcération a augmenté continuellement. Par contre, en Australie, à partir de 1972, on observe une chute très importante du volume d'incarcération (84,5 pour 100000 en 1972 : 63,7 en 1978). Ces observations jettent un doute incontournable sur le 
lien causal général et universel entre incarcération et criminalité que l'auteur évoque.

Très récemment, Fields (1990) a soumis les résultats d'une étude menée en Angleterre. Il y retenait un ensemble de variables socioéconomiques (police, démographie, niveau de consommation, etc.) dans des modèles de régression soigneusement ajustés. On constate parmi les observations importantes que la variation du volume d'arrestations est peu corrélée à celle de la criminalité connue. On relève également que ces variables jouent très différemment selon le type de crime analysé. Elles ne semblent pas permettre l'élaboration d'une théorie globale.

Nous avons déjà mentionné que les relations entre les variables socio-économiques et la criminalité changent selon le niveau d'agrégation spatiale. Ce fait a été bien établi notamment par R. N. Parker (1985). Mais elles changent également selon la période d'observation. Ceci signifie qu'une corrélation positive établie durant une période est susceptible de varier considérablement voire de disparaître ou de devenir négative à une autre période dans un même territoire. C'est la constatation faite par S. Mukherjee (1981) (chapitre 10). Or, la plupart des variables utilisées par l'auteur pour l'élaboration de sa théorie sont du type qui présente cette variabilité. Ce point est assez important, car il nous amène à interroger la durabilité de la théorie soumise, les faits et les observations sur lesquels elle repose étant incertains et fluctuants.

Dans cet échafaudage de propositions, on semble accorder une place relativement importante aux réactions d'autoprotection des victimes. Ceci vaut peut-être pour certains types de crimes, tels que le cambriolage ou le viol ; par contre, il est malaisé d'en percevoir la pertinence ou l'intérêt dans des incidents tels que l'incendie criminel ou le trafic de drogue ou le vol qualifié dans les lieux publics. Il faut également relever que l'autoprotection n'a pas seulement pour effet de réduire le nombre de certains types de crimes en limitant les occasions fournies aux prédateurs. Elle peut entraîner un déplacement des activités criminelles vers d'autres parties d'un territoire ou vers d'autres objectifs sur le même territoire ou même avoir pour effet pervers une augmentation du nombre de délits dans certains contextes. C'est, semble-t-il, ce qui s'est produit dans le cas des vols à main armée dans les banques à Montréal. Le fait de dégarnir les caisses, loin d'empêcher la vague de crimes, semble avoir eu pour effet de multiplier le nombre d'incidents en influençant la composition de la population de prédateurs. L. McPhetters (1976) signale que le durcissement des cibles peut avoir des effets négatifs ou imprévisibles, surtout lorsque la mesure 
n'est pas suivie d'un accroissement important de la probabilité d'arrestation. Donc, là encore, nous rencontrons une relation causale peu éclairée et peu éclairante.

Il nous serait loisible d'insister sur d'autres points du discours rencontré, mais ce qui précède nous suffit comme examen. La question se pose donc de savoir si on peut considérer comme une théorie formelle un ensemble de propositions reposant sur des relations inexistantes ou douteuses ou menacées d'obsolescence et de dislocations. Il est permis d'en douter. Il s'agit plutôt de variations paradigmatiques relativement sinon peu utiles et certainement pas nouvelles. Il y manque, pour nous résumer, des explications fondées sur des preuves et des généralisations étayées par des faits.

Mais il reste un point qui mérite considération. Est-il possible d'élaborer une théorie formelle pouvant expliquer les variations des taux des différents crimes enregistrés ou connus, avec ou sans victimes, contre les biens et contre les personnes ? Avant de répondre à la question posée, nous suggérons qu'on examine les faits suivants. Il faudrait d'abord avoir résolu les problèmes épineux de la mesure. Ceci est loin d'être fait. Par exemple, l'influence de l'autoprotection et des mesures de prévention retenue par l'auteur peut s'exercer d'une façon autre que celle mentionnée précédemment. En effet, en mobilisant l'attention et la sensibilité des victimes potentielles, ces mesures peuvent entraîner une augmentation du taux de signalement des crimes alors que leur nombre réel n'a peut-être pas changé de manière importante. Plusieurs projets de prévention ont déjà enregistré ce résultat paradoxal. Par ailleurs, il faut se rappeler que le taux de signalement fluctue selon les époques, le territoire, le type de crimes, les perceptions et les valeurs d'une population. Le problème de la mesure dans ce domaine n'est pas incontournable, mais il impose des contraintes importantes à la formalisation qui doivent être prises en compte dans chaque cas. Traiter le tout comme un champ unique nous apparaît comme un travers dont on aurait avantage à se libérer.

De plus, chaque type de criminalité évolue selon des dynamiques passablement différentes selon l'époque et le lieu, ainsi que nous l'avons déjà mentionné. On conçoit mal, dans ce contexte, l'intérêt et la possibilité d'émettre des formulations descriptives et explicatives globales. Enfin, nous n'avons aucune preuve du caractère réversible de ce que nous considérons comme les causes des phénomènes étudiés. Nous voulons dire par là que le retrait d'une de ces causes ne provoquera pas nécessairement une extinction du phénomène. Ainsi, il est tout à fait 
concevable que, dans un cas, une cause incitative mineure puisse déclencher une évolution en croissance rapide jusqu'au stade d'autoalimentation d'un phénomène, parce qu'une conjoncture ou une structure déjà présente, parfois invisible, contenait un potentiel multiplicateur. Dans ce cas, l'analyse du jeu des variables «explicatives» retenues dans des modèles de régression et même dans un système d'équations simultanées relève davantage d'une démarche interprétative qu'explicative. Tant que cette hypothèse, formulee en des termes falsifiables, n'est pas écartée, on ne peut accepter une théorie du type proposé.

Cette cause incitative n'est pas nécessairement perceptible et elle peut devenir rapidement inopérante une fois le processus en mouvement. Par ailleurs, elle n'aura pas systématiquement le même effet dans un autre contexte légèrement différent du précédent. Il est bien évident que ce point de vue interpelle notre manière d'expliquer, mais il est tout à fait admissible. Si on reconnaît que pour chaque type de crime des processus ou dynamiques différents peuvent exister selon le temps (courte ou longue période) et selon le lieu (l'unité géographique d'analyse), on doit renoncer à la formulation de théories globales et opter pour des propositions principalement descriptives et des théories à portée moyenne, adaptées à chaque type d'incident.

\section{BIBLIOGRAPHIE}

BILES, D. (1983), «Crime and Imprisonment ", British Journal of Criminology, vol. $23, n^{\circ} 2$, avril.

BLUMSTEIN, A. (1988), « Prison Populations : A System Out of Control ? ", in Crine and Justice. A Review of Research, Michael Tonry et Norval Morris (éd.), The University of Chicago Press, vol. 10.

BOWKER, L. H. (1981), "Crime and the Use of Prisons in the United States : A Time Series Analysis ", Crime and Delinquency, avril.

CHAMBliN, M. B. (1988), « Crime and Arrests : An Autoregressive Integrated Moving Average (ARIMA) Approach $*$, Joumal of Quantitative Criminology, vol. 4, $\mathrm{n}^{\circ} 3$.

CUSSON, M. (1990), Croissance et décroissance du crime, PUF.

FIELD, Simon (1990), Trends in Crime and Their Interpretation, Home Office Study $n^{\circ} 119$. 
GREENBERG, D. et KESSLER, R. (1982), «The Effect of Arrests on Crime : A Multivariate Panel Analysis $»$, Social Forces, vol. $60, \mathrm{n}^{\circ} 3$.

LIEBERSON, S. (1985), Making it Count. The Improvement of Social Research and Theory, University of Califomia Press.

McGUIRE, W. et SHEEHAN, R. (1983), * Relationships Between Crime Rates and Incarceration Rates : Further Analysis $"$, Journal of Research in Crime and Delinguency.

MCPHETTERS, L. R. (1976), "Criminal Behavior and the Gains from Crime », Criminology, vol. $14, \mathrm{n}^{\circ} 1$.

MUKHERJEE, S. K. (1981), Crime Trends in Twentieth-Century Australia, George Allen and Unwin, Pty. Ltd.

NCCD FOCUS (1988), The NCCD Prison Population Forecast : The Growing of Imprisonment in America, avril.

PARKER, R. N. (1985), * Aggregation, Ratio variables and Measurement Problems in Criminological Research ", Journal of Quantitative Criminology, vol. 1, $\mathrm{n}^{\circ} 3$.

PONTELL, H. N. (1979), «Deterrence, Theory vs Practice», Criminology, vol. 16, pp. 3-22.

TITTLE, C. R. et ROW, A. R. (1974), « Certainty of Arrest and Crime Rates : A Further Test of the Deterrence Hypothesis », Social Forces, vol. 52.

WELlFord, C. R. (1974), * Crime and the Police *, Criminology, vol. 12, $\mathrm{n}^{\circ} 2$.

Quelques tendances de la justice pénale canadienne (1984), rapport publié par le Groupe de recherche et de la statistique, Solliciteur général du Canada. 


\section{UNE THÉORIE DE L'ÉVOLUTION DE LA CRIMINALITÉ EST-ELLE POSSIBLE? \\ MAURICE CUSSON}

11 m'importe de donner la réplique à Daniel Élie sur quatre questions: 1) la détermination du niveau d'agrégation des phénomènes; 2) l'érosion de la peine ; 3) l'influence de la criminalité sur l'évolution pénale ; 4) la conception qu'il se fait de la théorie.

1) Mon collègue Élie me reproche de traiter la criminalité comme un bloc sans distinguer "des faits aussi divers que la fraude, le viol», etc. Il affirme aussi que le niveau d'agrégation spatiale que $\mathrm{j}$ 'ai retenu est trop élevé et il insiste (sans plus de précision) sur « la diversité des modes d'évolution d'une catégorie de crimes selon les zones étudiées». Il semble croire - à en juger par ce qu'il dit et écrit - qu'il existerait un principe méthodologique général qui stipulerait que le chercheur doit descendre au niveau d'agrégation le plus bas possible.

En réalité, la détermination du niveau d'agrégation des données est d'abord un problème pratique : elle dépend du problème que se pose le chercheur; et, ensuite, empirique : elle dépend de la configuration de ses données. Imaginons, par exemple, qu'un expert en analyse criminologique reçoive pour mission de découvrir les causes de la prolifération des vols de sac à main dans les rues du Vieux-Montréal. Il aura évidemment intérêt à se situer à un niveau très bas d'agrégation. Le problème que je me posais dans mon dernier livre était d'un tout autre ordre. J'avais été étonné par le parallélisme des séries chronologiques représentant l'évolution de plusieurs crimes bien mesurés entre 1960 et 1985 dans plusieurs pays occidentaux. Les courbes étaient tellement semblables que je ne pus m'empêcher de penser que des facteurs communs à tous les pays concernés étaient vraisemblablement à l'œuvre. Le fait que les courbes en question fluctuent parallèlement durant les mêmes années au Canada, aux États-Unis, en Angleterre, etc., est une donnée empirique qui saute aux yeux de quiconque regarde ces séries chronologiques. Nulle part dans son texte, Daniel Élie ne conteste ce fait. Il se contente d'affirmations générales comme: «le niveau d'agrégation spatiale est trop élevé ». Il l'est trop à son goût, pas au mien : $\mathrm{j}$ 'avais envie de rendre compte des caractéristiques de l'évolution de la criminalité communes à plusieurs pays.

S'agissant des types de délits, la question est à la fois empirique et théorique. C'est un fait d'observation que les taux d'homicides, de vols qualifiés et de cambriolages - pour ne parler que des crimes les mieux 
mesurés - ont des profils d'évolution très semblables entre 1960 et 1985. Alors pourquoi ne pas les traiter comme un tout? Il existe en outre plusieurs raisons théoriques bien connues qui nous aident à comprendre pourquoi des types de délits très variés fluctuent de conserve. On sait que la plupart des délinquants sont " polymorphes » : ils passent d'un type de délit à un autre au gré des occasions. La participation à la délinquance en général - et non à un type de délit - atteint un sommet durant la fin de l'adolescence et au début de l'âge adulte. Conséquence : une augmentation du pourcentage des 15-25 ans dans la population fera augmenter la criminalité toutes catégories confondues. Finalement, les théories du contrôle social et de la personnalité criminelle reposent toutes deux sur l'idée qu'il existe des facteurs qui génèrent chez certains individus une propension au crime assez indifférenciée, laquelle se traduit par une délinquance tous azimuts.

Contrairement à ce que M. Élie laisse entendre, il est évident que la science a pour ambition de se hisser au niveau de généralité le plus élevé possible. Elle vise aussi la parcimonie : rendre compte du plus grand nombre de faits possible avec le minimum de symboles. Une théorie qui réussit à expliquer un vaste ensemble d'observations est scientifiquement plus intéressante qu'une thérie qui ne vaut que pour une partie de cet ensemble. Pourquoi viser bas quand on peut viser haut?

2) On sait que j'ai défendu. dans le livre incriminé, la thèse selon laquelle la certitude de la peine a baissé dans plusieurs pays entre 1960 et 1975. Mon collègue s'appuie sur le fait que, au Canada, les taux d'incarcération par 100000 habitants sont stables depuis 1968 pour m'objecter qu'il n'y a pas eu d'érosion de la peine. Malheureusement, il passe complètement à côté de mon argument. J'ai écrit (p. 105) que pour estimer la certitude de la peine, il fallait rapporter le nombre de peines au nombre de crimes commis et non à la population. Quand la criminalité augmente à toute vitesse cependant que le niveau des peines reste à peu près stable, on est en droit de parler d'érosion : la probabilité qu'un délit soit puni baisse.

3) Dans le même ordre d'idées, mon collègue, citant Pontell, écrit ceci : « le taux de criminalité peut influencer plus fortement la certitude de la peine que la certitude de la peine influence le taux de criminalité ". Bien sûr ! Pourquoi enfoncer une porte ouverte ? Un lecteur le moindrement attentif verra que je consacre toute une section (pp. 110 à 119) de mon livre à expliquer comment l'augmentation de la criminalité fait baisser la probabilité de la punition. Je fais ensuite de ce phéno- 
mène un ingrédient essentiel de ma théorie formelle. J'écris notamment : « Les délits rapportés aux autorités devenant trop nombreux pour qu'ils soient tous traités, l'appareil judiciaire et pénal atteint un point de saturation. " Résultat: « le niveau de certitude de la peine baisse» (p. 139).

4) Au terme de son article, D. Élie avance - bien timidement, il faut le dire - ce qui semble être sa propre conception de l'analyse de la criminalité. "Il est tout à fait concevable, écrit-il, que, dans un cas une cause incitative mineure puisse déclencher une évolution en croissance rapide jusqu'au stade d'auto-alimentation d'un phénomène, parce qu'une conjoncture ou une structure déjà présente, parfois invisible, contenait un potentiel multiplicateur. " L'idée n'est pas très nouvelle : elle avait été défendue par Maruyama dans l'American Scientist en 1963 et elle avait été popularisée en criminologie par Wilkins en 1965 dans Social Deviance (voir p. 91).

Malheureusement, depuis 25 ans qu'elle est connue, cette proposition n'a pas fait faire de progrès fulgurants à la criminologie. La raison en est simple : elle conduit le chercheur qui voudrait s'en inspirer à l'impasse. Penser qu'une cause incitative " mineure ", " parfois invisible », pouvant « devenir rapidement inopérante » et n' ayant « pas systématiquement le même effet dans un autre contexte" puisse avoir une influence décisive sur la criminalité revient à dire qu'un deus ex machina insaisissable et changeant détient la clef du problème. Sa conciusion en découle logiquement; il faut, nous dit-il "renoncer à la formulation de théories globales » et opter pour « des théories de portée moyenne ».

Cependant, il affirme du même souffle que des processus différents peuvent exister selon le temps, selon le lieu et selon le type de délit, de telle sorte que des problèmes incontournables nous empêchent de passer d'un niveau d'agrégation inférieur à un niveau plus élevé. Si je comprends bien, nous sommes donc condamnés à rester au niveau microscopique. Dans ces conditions, comment pouvons-nous accéder au niveau moyen dont il nous parle?

Plusieurs problèmes que Daniel Élie nous présente comme insurmontables ont déjà été résolus et les solutions trouvées font d'ores et déjà partie des acquis des sciences sociales. Je me contenterai d'un exemple. Mon collègue observe que des corrélations observées dans un contexte ne se reproduisent pas nécessairement dans un autre. Le phénomène est connu depuis Le Suicide de Durkheim (1897) et les solutions théoriques au problème ne manquent pas. L'une d'elles repose 
justement sur la distinction proposée par Boudon entre théorie formelle et théorie au sens strict. La théorie formelle est tout le contraire d'un système de propositions présentées comme valables hors du temps et de l'espace. Elle est simplement une grille de lecture qui doit ensuite être appliquée aux phénomènes concrets que l'on se propose d'expliquer.

La possibilité qu'une cause n'ait pas l'effet prévu dans le modèle est reconnue par la notion même de théorie formelle. Le chercheur n'a pas à se surprendre si, dans un système ouvert et partiellement indéterminé, une variable extérieure au modèle intervient inopinément pour annuler ou modifier l'effet anticipé. C'est lors de la construction de la thérie au sens strict que l'on prend de telles variables en compte. Non, la recherche sur la criminalité n'est pas impossible. 